\title{
Methodik und klinische Bedeutung der quantitativen Bestimmung des $\alpha_{2}$-Serumhaptoglobins ${ }^{1}$
}

\author{
Von \\ R. Kluthe, R. Lumma, W. MÜLleR, H. MülleR \\ Aus der Medizinischen Universitäts-Poliklinik Freiburg i. Br. \\ (Direktor: Prof. Dr. H. Sarre) \\ und der Medizinischen Universitätsklinik Freiburg i. Br. \\ (Direktor: Prof. Dr. Dr. b.c. L. Heilmeyer)
}

(Der Schriftleitung zugegangen am 12. November 1962)

\begin{abstract}
Es wird eine einfache quantitative Haptoglobinbestimmungsmethode in Anlehnung an die sogenannte Aktivierungsmethode nach JAYLE beschrieben. Die Methode hat sich im Routinelaboratorium bewährt und ist auch im kleinen Laboratorium durchführbar. - Es wird über das Ergebnis quantitativer Haptoglobinbestimmung bei 63 Gesunden, 255 Patienten mit rheumatischen Erkrankungen, 34 Patienten mit Lebererkrankungen, 83 Patienten mit M. Boeck, 9 Patienten mit hämolytischen Erkrankungen und bei akuten und chronischen Nierenerkrankungen berichtet. Dabei wird gezeigt, daß die quantitative Haptoglobinbestimmung für die Beurteilung der entzündlichen Aktivität von rheumatischen Erkrankungen, Nierenerkrankungen und Lungenerkrankungen von Bedeutung ist. Auch für Diagnose und Verlaufsbeobachtung von Krankheitsbildern mit gesteigerter Hämolyse und zum Teil in Zusammenhang damit bei der Differentialdiagnose des Ikterus erwies sich die quantitative Haptoglobinbestimmung von Wert.
\end{abstract}

\begin{abstract}
A simple, quantitative method for haptoglobin determination, which depends on JAYLES so-called activation method, is described. The method is suitable for both routine and small laboratory analyses. The results of quantitative haptoglobin determinations are given for 63 healthy individuals, 255 with theumatic diseases, 34 with liver diseases, 83 with M. Boeck, 9 with hacmolytic diseases and for acute and chronic kidney diseases. It is thus shown that quantitative haptoglobin determination is significant for the diagnosis of inflammatory rheumatic, kidney and lung diseases. It is also useful for diagnosis and following the course of diseases involving increased haemolysis, and is thus an aid in the differential diagnosis of jaundice.
\end{abstract}

Die $\alpha_{2}$-Serumhaptoglobine machen beim Gesunden etwa $20 \% \operatorname{der} \alpha_{2}$-Globuline aus. Ausgedehnte Untersuchungen vor allem der JAYLEschen Schule haben gezeigt, daß auch unter pathologischen Bedingungen wie Entzündung bzw. Gewebszerfall eine gute Korrelation zwischen Haptoglobin- und $\alpha_{2}$-Globulinkonzentration besteht. (Vgl. 1, 2). Die einzigen bisher bekannten Ausnahmen sind die Nephrosen (3) und schwere septische Krankheitsbilder (2). Es hat sich erwiesen, daß die Aktivität chronisch-entzündlicher Prozesse mit der quantitativen Haptoglobinbestimmung besser erfaßt wird als durch die Bestimmung der Gesamt- $\alpha_{2}$-Fraktion. Darüber hinaus ist die quantitative Haptoglobinbestimmung für die Beurteilung einer gesteigerten Hämolyse gut geeignet. Hierbei kommt es durch die Eigenschaft des Haptoglobins ( $\mathrm{Hp}$ ), Hämoglobin $(\mathrm{Hb}) \mathrm{zu}$ binden, zur vermehrten Bildung eines Haptoglobin-HämoglobinKomplexes ( $\mathrm{HpHb})$. Der Komplex $\mathrm{HpHb}$ wandert mit einer Halbwertzeit von etwa einer halben Stunde in das RES ab. Die quantitative.Hp-Bestimmung stellt unter Berücksichtigung dieser Tatsache einen guten Indikator für den Hämolysegrad dar.

1) Unterstützt durch eine Sachbeihilfe der Deutschen Forschungsgemeinschaft.
Zur Bestimmung des $\alpha_{2}$-Haptoglobins stehen folgende Methoden zur Verfügung:

1. Die von JayLe (4) entwickelte Peroxydasimetrie.

2. Die von Laurell und Nymann (5) angewandte direkte Messung der Hämoglobinkapazität des Serums.

3. Eine von Connell und Smithies (6) entwickelte Modifikation der Methode nach JAYLE.

Alle benutzen die Eigenschaft des Haptoglobins, mit Hämoglobin eine stabile Verbindung einzugehen. JAYLE (4) mißt die Peroxydaseaktivität des in vitro gebildeten Haptoglobin-Pferdehämoglobinkomplexes. Connell und Smithies (6), bestimmen die Peroxydaseaktivität des Haptoglobin-Humanhämoglobinkomplexes. Laurell und NymanN (5) testen durch Zugabe von steigenden Mengen Hämoglobin zu verschiedenen Proben des gleichen Serums, wann in der Papierelektrophorese neben der $\mathrm{HpHb}$-Bande langsamer wanderndes freies Hämoglobin auftritt. Aus dieser Grenzkonzentration ergibt sich die hämoglobinbindende Kapazität des Serums, die dem Eiweißanteil des Haptoglobins entspricht, die sog. Haemoglobin Binding Capacity ( $\mathrm{HbBC}$ ). Nach unseren Erfahrungen ist das JAYLEsche Prinzip für klinische Bedürfnisse am besten geeignet. Wir haben diese sog. Aktivierungsmethode 
(méthode d'activation), modifiziert, um ihre Durchführung auch im kleinen Laboratorium möglich $\mathrm{zu}$ machen.

\section{A. Methodik}

Prinzip (nach JAYLE): Das im Serum enthaltene Haptoglobin wird durch Zugabe eines Überschusses von Pferde- oder Human-Hämoglobin in den HpHb-Komplex übergeführt. Dieser hat eine starke peroxydatische Wirkung im sauren Milieu, wogegen die Peroxydaseaktivität des wechselnden $\mathrm{Hb}$-Uberschusses hier nicht in Erscheinung tritt. Je nach der peroxydatischen Aktivität des HpHb-Komplexes wird Äthylhydroperoxyd in Äthylalkohol und atomaren Sauerstoff gespalten. Ein Atom Sauerstoff nimmt zwei Elektronen auf, die es von den im Substrat befindlichen Jodionen erhält. Letztere werden dabei zu elementarem Jod oxydiert und durch Jodometrie bestimmt.

\section{Reagenzien}

\section{1. $8 \times 10^{-4}-m$-Pferdebämoglobinlösung:}

Zitriertes Pferdeblut wird zentrifugiert, das Serum abgehebert und die Zellen $3 \times$ mit 0,15-m-NaCl-Lösung gewaschen. Zur Gewinnung der Hämoglobinlösung. werden $14 \mathrm{ml}$ Erythrozytensediment in cinen $100 \mathrm{~m} / \mathrm{Meßkolben}$ gebracht und mit $\mathrm{H}_{2} \mathrm{O}$ aufgefüllt. Ein Abzentrifugieren der Stromata kann unterbleiben. $1 \mathrm{~m} /$ Hämoglobinlösung wird nach vorsichtigem Schütteln entnommen und die Hämoglobinkonzentration nach ZIJLSTRA (7) bestimmt. Durch Zusatz von Kaliumferricyanid wird das Hämoglobin in Cyanmethämoglobin umgewandelt. Die Cyanmethämoglobin-Konzentration ermittelt man durch Messung der Extinktion im Spektralphotometer bei $545 \mathrm{~m} \mu: 1 \mathrm{~m} l$ Hb-Lösung wird mittels einer geeichten Pipette in einen $100 \mathrm{~m} l$ Meßkolben gebracht, der etwa $20 \mathrm{~m} / \mathrm{H}_{2} \mathrm{O}$ enthält. Die so verdünnte $\mathrm{Hb}$ Lösung wird mit 2 Tropfen 10 proz. $\mathrm{K}_{3}\left[\mathrm{Fe}\left(\mathrm{CN}_{8}\right)\right]$-Lösung versetzt. Nach 5 ' werden $2,5 \mathrm{~m} l 0,1 \mathrm{prcz}$. NaCN-Lösung hinzugefügt und mit $\mathrm{H}_{2} \mathrm{O}$ aufgefüllt. Messung im Spektralphotometer bei $545 \mathrm{~m} \mu$. Bei einer Schichtdicke von $d=10 \mathrm{~mm}$ gilt: $\mathrm{C}=\mathrm{E} \times 14,5(\mathrm{C}=$ Hämoglobinkonzentration der Probe in $\mathrm{g} / 100 \mathrm{ml}, \mathrm{E}=$ Extinktion.) Nun wird die Konzentration der Hämoglobin-Lösung auf einen $\mathrm{Hb}-\mathrm{Gehalt}$ von $34 \mathrm{~g} / \mathrm{l}$ eingestellt. Diese Lösung stellt die Stammlösung dar $(\mathrm{m} / 2000)$, die bei Gebrauch mit $\mathrm{H}_{2} \mathrm{O}$ 1:40 verdünnt wird $(\mathrm{m} / 80000)$. Die Stammlösung ist eingefroren monatelang haltbar. Die Gebrauchslösung muß täglich neu angesetzt werden.

\section{0,1-n-Ätbylbjdroperoxyd}

$0-1-n$-Åthylhydroperoxydlösung wird ein- bị zweimal pro Woche durch Verdünnen aus einer konzentrierten Lösung ${ }^{1}$ ) hergestellt. (Aufbewahrung in brauner Flasche bei $+2^{\circ}$ bis $+8^{\circ}$.) Die Bestimmung der Normalität der konzentriertẹn Äthylperoxydlösung wird nach der Methode von Wrbout, van LEUWWeN und van der WAL (8) vorgenommen: In einem $300 \mathrm{~m}$-Erlenmeyerkolben, der durch ein Uberleitungstoht mit einem Becherglas, das $\mathrm{H}_{2} \mathrm{O}$ enthält, verbunden ist, gibt man $1,5 \mathrm{~g} \mathrm{NaHCO}_{3}$ und $20 \mathrm{ml}$ Eisessig. Ein kleineres Gefäß (etwa $1 \mathrm{~m} l$ fassend), in dem sich $0,1 \mathrm{~m} l$ der zu prüfenden Âthylperoxydlösung befinden, wird aufrecht in den Erlenmeyerkolben' gestellt. $5 \mathrm{ml}$ einer 40 proz. wäßrigen Kaliumjodidlösung werden zum Inhalt des Erlenmeyerkolbens hinzugefügt, der dann an das Uberleitungsrohr angeschlossen wird. Wenn die $\mathrm{CO}_{2}$-Entwicklung beendet ist (nach' etwa $2^{\prime}$ ), sollte das Reaktionsgemisch im Erlenmeyerkolben farblos sein. Jetzt wird durch Schüttcln das Äthylperoxyd mit dem Inhalt des Erlenmeyerkolbens gemischt. Die Reduktion des Äthylperoxyds dauert mindestens $10^{\prime}$. Die Reaktion soll im Dunkeln ablaufen. An-

1) Firma Ferrosan, Malmö, Schweden. schließend füllt man $50 \mathrm{~m} / \mathrm{H}_{2} \mathrm{O}$ ein und titriert mit $0,1-n \mathrm{Na}_{2}$ $\mathrm{S}_{2} \mathrm{O}_{3}$-Lösung. 2proz. Stärkelösung dient als Indikator.

3. 0,01-n-Jodlösung

$20-25 \mathrm{~g}$ jodatfreies Kaliumjodid werden im $1000 \mathrm{~m} / \mathrm{Meßkolben}$ in etwa $40 \mathrm{~m} / \mathrm{H}_{2} \mathrm{O}$ gelöst und $12,7 \mathrm{~g}$ Jod hinzugegeben. Der verschlossene Kolben wird solange geschüttelt bis alles Jod in Lösung gegangen ist und mit $\mathrm{H}_{2} \mathrm{O}$ bis zur Marke aufgefüllt. Die erhaltene Lösung ist dann etwa $0,1-n$. Der Faktor wird mit $0,1-n$-Natriumthiosulfatlösung bestimmt und sollte regelmäßig kontrolliert werden. Aus der Stammlösung wird täglich eine 0,01-n Gebrauchslösung hergestellt.

4. 0,1-m-Kaliumjodidlösung

$16,6 \mathrm{~g} \mathrm{KJ}$ ad $1000 \mathrm{~m} / \mathrm{H}_{2} \mathrm{O}$.

5. 0,01-n-Natriumthicsulfatlösung

aus einer $0,1-n$-Stammlösung durch Verdünnen täglich frisch zubereitet.

\section{Acetatpuffer $p H=4,4$}

$13,6 \mathrm{~g} \mathrm{Na}$-Acetat und $11,0 \mathrm{~m} /$ Eisessig werden mit $\mathrm{H}_{2} \mathrm{O}$ auf $1 l$ aufgefüllt. Mit einem pH-Meter wird die Wasserstoffionenkonzentration gemessen und wenn nötig mit Eisessig oder Natronlauge auf $\mathrm{pH}=4,4$ eingestellt. Bei $+2^{\circ}$ b:s $+8^{\circ}$ aufbewahren.

\section{2proz. Stärkelösung}

\section{Durchführung}

$0,02 \mathrm{ml}$ Serum werden in einem $50 \mathrm{ml}$ Becherglas mit $0,1 \mathrm{ml}$ Pferdehämoglobinlösung $8 \times 10^{-4} \mathrm{~m}$ gemischt. Dann werden $3 \mathrm{~m} l$ 0,1-m-Kaliumjodidlösung hinzugegeben. In einem zweiten Becherglase werden $10 \mathrm{ml}$ Azetatpuffer ( $\mathrm{pH}=4,4)$, mit $1,0 \mathrm{~m} / 0,1-n$-Äthylhydroperoxydlösung und $0,5 \mathrm{ml} 0,01-n$-Jodlösung zusammengegeben. Der Inhalt des zweiten Becherglases wird in Becherglas 1 übergeführt und genau nach $5^{\prime}$ mit einer 0,01- $n$-Natriumthiosulfatlösung titriert. Von dem erhaltenen Wert wird der Titrationswert einer Blindbestimmung ohne Serum abgezogen. Das resultierende Volumen 0,01- $n$-Natriumthiosulfat (,wahrerVerbrauch “) wird mit dem Faktor 207 (NyMANN (1)) und einem Temperatur-Korrekturfaktor (Abb. 1) multipliziert. Die

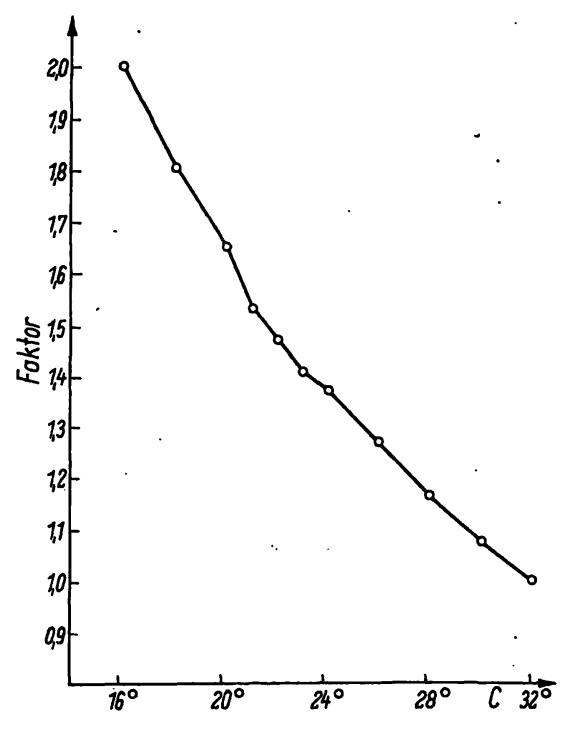

Abb. 1.

Experimentell ermittelter Tempcraturfaktor für verschiedene Arbeitstemperaturen (Faktor bei $32^{\circ}=1,0$ ) 
Originalmethode nach JAYLE sieht die Bestimmung bei $32^{\circ}$ vor. Durch den Temperatur-Korrekturfaktor ist eine Umrechnung auf jeweilige Arbeitstemperatur möglich. Es muß darauf geachtet werden, daß sämtliche Lösungen die gleiche Temperatur aufweisen. Es ist notwendig, bei Serienbestimmungen zur Kontrolle ein Testserum mit mittlerem Hp-Gehalt mitlaufen zu lassen.

\section{Fehlermöglichkeiten}

Der methodische Fehler ist relativ gering. In unserem Laboratorium ermittelten wir einen Fehler von $\pm 1,5 \%$ bei $32^{\circ}(11 \mathrm{Be}-$ stimmungen) und $2,0 \%$ bei $21^{\circ}$ (10 Bestimmungen) in einem Serum von $102 \mathrm{mg} \%$ HbBC. Bei hämolytischen Seren (Abb. 2) bildet sich je nach Gehalt der Serumprobe an Humanhämoglobin der Komplex Hp-Human-Hb, dessen Peroxydaseaktivität nur etwa halb so groß ist, wie die des Komplexes HpPferde-Hb. Es empfiehlt sich also bei hämolytischen Seren, die Bestimmung mit Human-Hb wie oben. Das Ergebnis muß dann mit dem Faktor 2,08 multipliziert werden, wodurch der methodische Fehler bei Bestimmung mit Human-Hb größer wird als bei der Bestimmung mit Pferdehämoglobin.

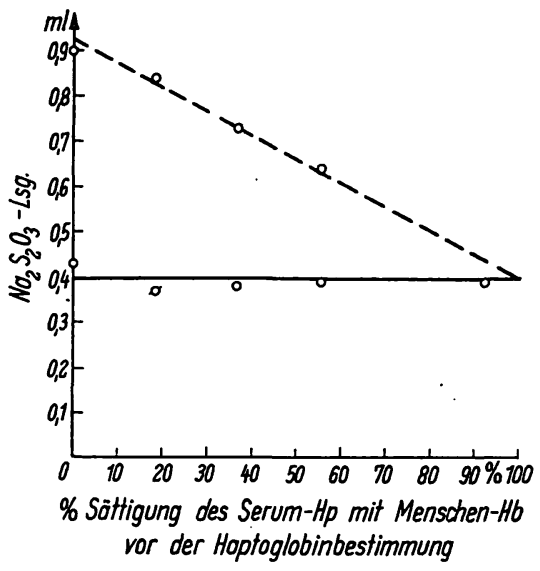

Abb. 2.

Verbrauch an 0,01-n Natriumthiosulfat in Anwesenheit von steigenden Mengen Human-Hb.

-Bestimmung mit Human-Hb.

- - - Bestimmung mit Pferde-Hb.

(in Anlehnung an NyMANN (1))

Werden bei derTitration mehrals $1,7 \mathrm{~m} / \mathrm{Na}_{2} \mathrm{~S}_{2} \mathrm{O}_{3}$-Lösung verbraucht, muß die Hp-Bestimmung mit 1:1 verdünntem Serum wiederholt werden. Bei niedrigen Titrationswerten $(<0,1 \mathrm{~m} l)$ wiederholen wir die Bestimmung mit $0,05 \mathrm{~m} l$ Serum bei $32^{\circ}$ und wenn dann kein oder praktisch kein Thiosulfatverbrauch vorliegt, wird eine immunologische Bestimmung von Haptoglobin mit Hilfe eines eigenen vom Kaninchen gewonnenen spezifischen Anti-Haptoglobin-Serums vorgenommen. Mittels dieser neuen Methode, die 20 fach empfindlicher ist als die Peroxydasemethode, lassen sich noch Spuren von $\mathrm{Hp}$ nachweisen. Dies ist vor allem für die $\mathrm{Ab}-$ grenzung der Ahaptoglobinämie wichtig, mit deren Diagnose man nach unseren Erfahrungen mit der quantitativen peroxydasimetrischen und immunologischen Hp-Bestimmung vorsichtig sein muß. Wir haben oft noch immunologisch Haptoglobin nachweisen können, wenn peroxydasimetrisch nichts nachweisbar war (KLUTHE und Mitarbeiter (9)).

\section{Berechnung}

Wahrer Verbrauch $m / 0,01-n$-Natriumthiosulfat $\times 207$ $($ NYMANN $(1)) \times$ Temperaturfaktor (Abb. 1) $=\mathrm{mg} \%$ $\mathrm{HbBC}=$ Proteinanteil des Haptoglobins.

Aus Griinden der besseren Vergleichbarkeit der Ergebnisse sind wir der Ansicht, daß man den Gehalt an Haptoglobin in $\mathrm{mg} \%$
$\mathrm{HbBC}$ angeben sollte. Diese Größe entspricht dem Eiweißanteil dieses Mucoproteins und zeigt eine enge Korrelation zur Peroxydaseaktivität (1). Man hat dann auch den Vorteil, daß sich die gewonnene Größe, der Eiweißanteil des Haptoglobins, leichter mit den papierelektrophoretischen u. a. Eiweißbestimmungen im Serum vergleichen läßt. In unseren sämtlichen sich auf $\mathrm{Hp}$ beziehenden Publikationen sind wir in dieser Weise verfahren.

\section{Erkerankungen des rbeumatischen Formenkreises}

Im Rahmen von Untersuchungen über das Verhalten verschiedener Entzündungsindikatoren wurde von LACAPERE u. Mitarbeitern (10) auch das Haptoglobin quantitativ bestimmt. Es ergab sich ähnlich wie früher bei anderen entzündlichen Erkrankungen eine gute Brauchbarkeit für die Aktivitätsdiagnostik, wie orientierende Arbeiten der Jayleschen Schule $(11,18)$ schon früher und AlLISON und Blumberg (12) demonstrierten. Die Bedeutung von Hp-Bestimmungen bei chronischer Polyarthritis ist heute noch umstritten. Vor allem durch die Einteilung der chronischen Polyarthritis in eine progredient verlaufende und eine zyklische Form ergaben sich neue Gesichtspunkte, die eine systematische Analyse des Verhaltens von Hp (und anderer Serumfaktoren) notwendig erscheinen ließen. Wir haben daher im Rahmen solcher Untersuchungen u. a. auch das Serumhaptoglobin quantitativ bestimmt. Über erste Befunde wurde von uns bereits an anderer Stelle berichtet $(13,14)$. Nach Erweiterung unserer Untersuchungen ergibt sich folgendes Bild: (Tab. 1). Man sieht, daß bei kontinuierlicher progredienter chronischer Polyarthritis und zyklischer chronischer Polyarthritis ebenso wie beim M. Bechterew in einem hohen Prozentsatz Hp erhöht ist. Die Hp-Erhöhung geht, wie sich zeigen ließ, weitgehend parallel der klinischen Aktivität (14). Die quantitative Hp-Bestimmung ist demnach bei beiden Formen. der chronischen Polyathritis für die Aktivitätsdiagnostik gut geeignet. Bei Arthrosen ohne entzündliche Komplikation findet man mit 90,7 als Mittelwert einen Wert, der mit unserem an 63 Gesunden gefundenen Wert gut übereinstimmt.

Tab. 1

Haptoglobin bei Erkrankungen des theumatischen Formenkreises.

\begin{tabular}{cccc}
\hline Diagnose & $\begin{array}{c}\text { Zahl der } \\
\text { Patienten }\end{array}$ & $\begin{array}{c}\text { davon } \\
\text { erhöht }\end{array}$ & $\begin{array}{c}\text { Mittelwert } \\
\pm 2 \sigma \mathrm{mg} \% \mathrm{HbBC}\end{array}$ \\
\hline
\end{tabular}

Kontrollen

63

-

$86,1 \pm 49,2$

Kontinuierlich progre-

diente chron.

Polyarthritis

100

$90 \%$

$254,4 \pm 179,6$

Zykl. chron.

Polyarthritis

92

$83 \%$

$227,0 \pm 206,9$

M. Bechterew

32

$87 \%$

$239,0 \pm 164,2$

Arthrosen m. entz.

Komplikationen

10

$70 \%$

$163,5 \pm 97,5$

Arthrosen ohne entz.

Komplikationen
$21 \quad 0 \%$ 


\section{Nierenerkrankungen}

Wie wir bereits früher (15) zeigen konnten, ist bei Nierenerkrankungen das Serumhaptoglobin häufig erhöht. Bei akuter Glomerulonephritis und subakuter Glomerulonephritis findet man durchweg hohe, bei chronischer Glomerulonephritis z. T. auch normale und erniedrigte Werte. Nach den bisherigen Verlaufsbeobachtungen sind die Fälle mit Hp-Erhöhungen die progredient verlaufenden mit entzündlicher Aktivität (16). Hp-Erniedrigungen im Spätstadium sind wohl auf den bei Urämie gesteigerten Blutumsatz oder auf eine zusätzliche Leberschädigung zurückzuführen. Bei Fällen mit Resthypertonie ist $\mathrm{Hp}$ durchweg normal. Diese Beobachtung ist von Bedeutung, da man so ohne lange Verlaufsbeobachtung mit Clearance und Nierenpunktion u. U. über die Progredienz des Leidens etwas aussagen kann. Auch bei der chronischen Nephritis mit nephrotischer Verlaufsform findet sich Hp immer erhöht. An Hand des Haptoglobins läßt sich hier der Therapieerfolg gut absehen. Für die Pyelonephritis ergibt sich ähnliches. Auch bei diesem Krankheitsbild ist die Haptoglobinerhöhung als Ausdruck einer noch floriden Entzündung anzusehen. Dieser Befund ist vor allem deswegen von Bedeutung, weil uns die Blutsenkungsreaktion als sonst empfindlicher Indiktaor bei den Fällen von chronischer Pyelonephritis sehr häufig im Stich läßt (16). Eine differentialdiagnostische Abtrennung von Glomerulonephritis und Pyelonephritis ist durch die quantitative Haptoglobinbestimmung nicht möglich.

\section{Tuberkulose und Morbus Boeck}

Auf die klinische Bedeutung der quantitativen Haptoglobinbestimmung bei Lungentuberkulose wurde bereits mehrfach hingewiesen. $(17,18)$. Von Interesse ist, $\mathrm{da} B$ eine Korrelation zwischen der (einfachen und meist als Indikator für die Aktivität des Prozesses bei Tuberkulose angewandten) BSG und dem Haptoglobinspiegel nur im Bereich hochpathologischer Werte besteht. Bei niedriger BSG erwies sich die quantitative Bestimmung von Haptoglobin für die Beurteilung des Krankheitsprozesses als besonders wertvoll. KourILSKY und Mitarbeiter (19) fanden beim Vergleich der klinischen Aktivität von 83 Patienten mit Lungentuberkulose, daß das Hp den Krankheitsprozeß besser widerspiegelt als die BSG. Während die Hp-Bestimmung in 33 Fällen dem klinischen Bild besser als die BSG entsprach, war dies umgekehrt nur in 5 Fällen zu beobachten. In der Hälfte der Fälle (52\%) war der Ausfall der Reaktionen konkordant.

Die quantitative Hp-Bestimmung ist also bei der Lungentuberkulose vor allem für die Feindiagnostik bei diskreten Krankheitszeichen und im Verdachtsfall angezeigt. $\mathrm{Da}$ wir beim Morbus Boeck ungleich häufiger normale BSG-Werte finden, wenn klinisch eine Aktivität der Erkrankung angenommen werden muß, haben wir in Zusammenarbeit mit WURM untersucht, ob auch hier ähnlich wie bei der Lungentuberkulose durch die Bestimmung des Serumhaptoglobins eine Verbesserung der Aktivitätsdiagnostik erreicht werden kann. Über vorläufig erhobene Befunde wurde bereits in einem anderen Rahmen berichtet (20). Auch hier fand sich eine deutliche Überlegenheit der quantitativen $\mathrm{Hp}$ Bestimmung gegenüber der Blutsenkung. Die HpBestimmung lieferte in 14 Fällen von 83 alleinige Hinweise auf das Vorliegen einer Aktivität des Krankheitsprozesses. Diese Untersuchungen werden zur Zeit noch weitergeführt und an einem größeren Krankengut mit Verlaufsbeobachtungen überprüft.

\section{Lebererkrankungen}

Bei Lebererkrankungen findet man in vielen Fällen erniedrigte Serumhaptoglobinspiegel, vor allem bei chronischen Erkrankungen (1, 21). Über das Verhalten bei akuten Krankheitsprozessen liegen unterschiedliche Befunde vor. Während JAYLE und VAlLIN (21) bei infektiöser Hepatitis im Durchschnitt erniedrigte HpSpiegel fanden, bewegt sich dieser nach NyMANN (1); bei größeren individuellen Schwankungen im Normbereich. In eigenen Untersuchungen an einem kleineren Material (Tab. 2) fanden wir bei akuter Hepatitis, subakuter Hepatitis und Leberzirrhose einen signifikant niedrigeren Mittelwert im Vergleich zu 63 Normalpersonen. Bei Ikterus durch Cholangitis-Cholangiolitis war der Spiegel in den 4 untersuchten Fällen erhöht. Unsere Befunde stimmen mit den von Owen (22) publizierten Daten gut überein. Man kann also der quantitativen Hp-Bestimmung eine Bedeutung für die Differentialdiagnose des Ikterus zumessen. Hohe Hp-Spiegel sprechen gegen einen Leberzellschaden und eine Hämolyse. Bei niedrigen Titern oder Fehlen des Haptoglobins kann sowohl ein hepatischer Ikterus als auch eine Hämolyse vorliegen. Mit der Diagnose einer Ahaptoglobinämie sowohl beim Gesunden als auch beim Kranken sollte man zurückhaltend sein (s. o.). Es ist dies in erster Linie eine Frage der Empfindlichkeit der Nachweismethode. Wir haben häufig mit der 20 fach empfindlicheren immunologischen Methode noch Haptoglobin

Tab. 2

Haptoglobin bei Lebererkrankungen

\begin{tabular}{|c|c|c|c|c|}
\hline Diagnose & $\begin{array}{l}\text { An- } \\
\text { zahl }\end{array}$ & $\begin{array}{c}\text { Mittelwert } \\
\text { (mg\% } \\
\text { HbBC) }\end{array}$ & $\begin{array}{l}\text { Standard- } \\
\text { abweichung }\end{array}$ & Signifikanz \\
\hline Kontrollen & 63 & 86,1 & 24,6 & - \\
\hline Akute Hepatitis & 13 & 57,2 & 45,2 & $0,02>P<0,03$ \\
\hline $\begin{array}{l}\text { Subakute-chron. } \\
\text { Hepatitis }\end{array}$ & 6 & 61,5 & 21,3 & $P<0,01$ \\
\hline Leberzirrhose & 8 & 48,9 & 46,5 & $0,02>P<0,03$ \\
\hline $\begin{array}{l}\text { Cholangitis- } \\
\text { Cholangiolitis }\end{array}$ & 4 & 213,5 & 39,5 & $P<0,001$ \\
\hline $\begin{array}{l}\text { Posthepatit. } \\
\text { Hyperbilirubin- } \\
\text { ämie }\end{array}$ & 2 & 86,0 & - & - \\
\hline Leberkarzinom & 1 & 153,0 & - & - \\
\hline
\end{tabular}


nachweisen können, wenn die Peroydasemethode eine Ahaptoglobinämie anzeigte.

Die Pathogenese der niedrigen Hp-Titer bei Lebererkrankungen ist noch unklar. Manche Autoren diskutieren eine Störung der Hp-Synthese in der Leber, dem wahrscheinlichen Bildungsort, andere wiederum vermuten eine Mikrohämolyse. Auch wurde eine Hemmung der Peroxydaseaktivität des Komplexes $\mathrm{HpHb}$ sowie auch eine Störung bei der Bildung des Komplexes durch Milieufaktoren (Gallensäuren usw.) diskutiert. Letzteres ist nach den Ergebnissen unserer bisherigen immunologischen Untersuchungen wenig wahrscheinlich. Eingehende Untersuchungen mit Bestimmung der Überlebenszeit der Erythrozyten sind im Gange.

\section{Das Verbalten des Haptoglobins bei gesteigerter Hämolyse}

Vor allem durch die Arbeiten von Nymann (1) wurde auf die Bedeutung von $\mathrm{Hp}$ für den Eisenstoffwechsel hingewiesen. Eine der wesentlichen bis heute bekannten Funktionen besteht in der Bindung von freiem Hämoglobin, was den Eisenverlust durch die Niere verhindert. Der Komplex $\mathrm{HpHb}$ wird erwiesenermaßen nicht glomerulär filtriert und auch nicht tubulär ausgeschieden. Erst nach Erschöpfung der Hämoglobinbindungskapazität des Serums kommt es zur Hämoglobinurie. Nach den bis heute vorliegenden Untersuchungen ist die Nierenschwelle für $\mathrm{Hb}$ höchstwahrscheinlich durch den Hp-Gehalt des Serums vorgetäuscht. Diese Frage ist jedoch noch nicht genügend abgeklärt (Übersicht bei SCHUBOthe u. KähLER (23)).

Bindet man durch Hämoglobininjektion das zirkulierende Haptoglobin, so kommt es bei einer erneuten Hämoglobininjektion zur Hämoglobinurie. Für Mikrohämolysen ist die quantitative Hp-Bestimmung ein feineres $\mathrm{Ma} \dot{B}$ als die Bestimmung des Hämoglobinspiegels, da der Komplex $\mathrm{HpHb}$ mit einer Halbwertzeit von etwa $30^{\prime}$ in das RES abwandert. Bei Anwendung der üblichen Hämoglobinbestimmungen kann sich also eine Hämolyse unter Umständen dem Nachweis entziehen. Die quantitative Hp-Bestimmung erlaubt hier einen exakten Nachweis, wie wir am Beispiel folgender Bestimmungen vor und nach extrakorporaler Hämodialyse zeigen.

\section{a) Haptoglobin bei extrakorporaler Dialyse}

Es ist für die Beurteilung und Vermeidung von Komplikationen von Bedeutung, ob in dem einen oder anderen Fall von extrakorporaler Hämodialyse eine Hämolyse auftritt. Die Messung des Hämoglobins im Serum hat sich für diese Fragestellung methodisch als ungenügend erwiesen. Wir haben daher bei 9 Fällen von extrakorporaler Hämodialyse vor und nach der „Künstlichen Niere" Hp bestimmt (Abb. 3). Man sieht, $\mathrm{da} B$ der Spiegel, wenn auch unterschiedlich, in allen Fällen absinkt. Unter Berücksichtigung des Plasmavolumens läßt sich die freigewordene Hämoglobinmenge gut errechnen ( $1 \mathrm{mg} \mathrm{Hp}$ [Eiweißanteil] bindet

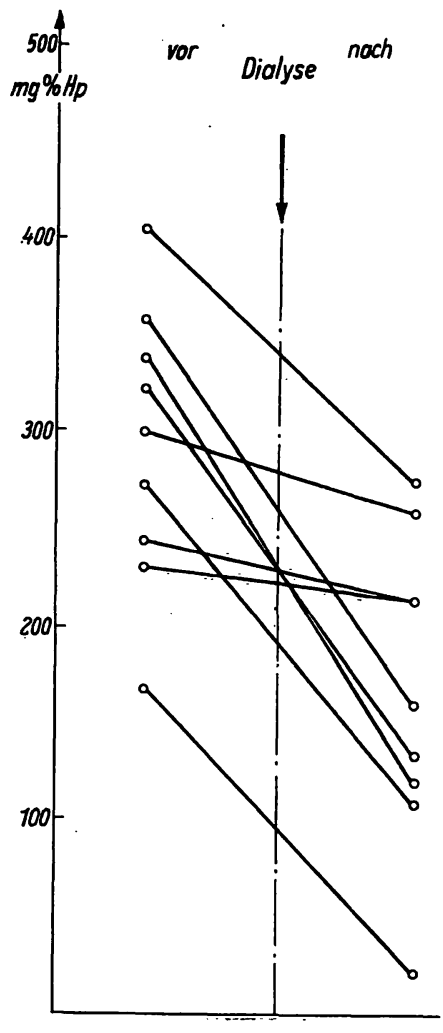

Abb. 3.

Der Hp-Spiegel zu Beginn und am Ende von 9 extrakorporalen Hämodialysen bei akuter Anurie unterschiedlicher Ätiologie.

$1 \mathrm{mg} \mathrm{Hb}$ und schleust dieses höchstwahrscheinlich aus der Blutbahn in das RES).

\section{b). Haptoglobin bei bämolytischen Erkrankungen}

Auch bei hämolytischen Etkrankungen ist die quantitative Hp-Bestimmung von Nutzen. Tabelle 3 zeigt die

$$
\text { Tab. } 3
$$

Haptoglobin und Halblebenszeit mit Cr 51 markierten Erythrozyten bei hämolytischen Erkrankungen

\begin{tabular}{|c|c|c|c|}
\hline Name & Diagnose & $\begin{array}{c}\text { Haptoglobin- } \\
\text { gehalt } \\
\text { (mg\% } \\
\text { HbBC) }\end{array}$ & $\begin{array}{l}\text { Halblebens- } \\
\text { zeit von Cr } \\
\text { 51-markiet- } \\
\text { ten Ery- } \\
\text { throzyten }\end{array}$ \\
\hline $\begin{array}{l}\text { 1. S. M. } \\
\text { 2. H. L. } \\
\text { 3. M. H. } \\
\text { 4. P. F. } \\
\text { 5. K. A. } \\
\text { 6. F. E. } \\
\text { 7. G. L. } \\
\text { 8. M. I. } \\
\text { 9. M. J. }\end{array}$ & $\begin{array}{l}\text { Kongenitale } \\
\text { hämolyt. Anämie } \\
\text { Kongenitale } \\
\text { hämolyt. Anämie } \\
\text { paroxysmale nächtliche } \\
\text { Hämoglobinurie } \\
\text { Autoimmunhämolyt. } \\
\text { Anämie } \\
\text { Autoimmunhämolyt. } \\
\text { Anämie } \\
\text { Autoimmunhämolyt. } \\
\text { Anämie } \\
\text { Autoimmunhämolyt. } \\
\text { Anämie } \\
\text { Autoimmunhämolyt. . } \\
\text { Anämie } \\
\text { Autoimmunhämolyt. } \\
\text { Anämie }\end{array}$ & $\begin{array}{r}0 \mathrm{mg} \% \\
30 \mathrm{mg} \% \\
0 \mathrm{mg} \% \\
0 \mathrm{mg} \%\end{array}$ & $\begin{array}{l}13 \text { Tage } \\
16 \text { Tage } \\
7 \text { Tage } \\
3 \text { Tage } \\
6 \text { Tage } \\
7 \text { Tage }\end{array}$ \\
\hline
\end{tabular}


Haptoglobinwerte bei verschiedenen Fällen von gesteigerter Hämolyse bei kongenitaler und erworbener hämolytischer Anämie. Wir finden also zum Teil kein Haptoglobin in diesen Seren, zum Teil ist das Haptoglobin deutlich erniedrigt. Die Befunde stimmen recht gut mit den von NymanN (24), (mit Hilfe der direkten Messung der Hämoglobinbindungskapazität) früher gemachten Beobachtungen überein, $\mathrm{da} B$ bei einer Verkürzung der Halblebenszeit Cr 51-markierter Erythro- zyten auf unter 17 Tage kein Haptoglobin im Sẻrum nachweisbar ist. Untersuchungen mit immunologischer Haptoglobinbestimmung sind z. Z. im Gange.

Die Untcrsuchungen wurden in Seren von Patienten der Med. Univ.-Klinik Freiburg durchgeführt, die uns freundlicherweise von Herrn Professor Schubothe überlassen wurden. Für die Ergebnisse der Halblebenszeitbestimmung der Erytbrozyten durch $\mathrm{Cr}$ 51-Markierung bedanken wir uns herzlich bei Herrn Dr. Heimper und Herrn Professor Kerdering, Med. Univ.Klinik Freiburg.

\section{Literatur}

1. Nrmann, M. Scand.J. Clin.Laborat. Invest. Suppl. 11, 39 (1959).2. Schumacher, G., Habilitationsschrift Tübingen (1962). 3. Jayle, M. F., G. Ligrue, und G. Boussizr, Presse méd. Paris. 62, 1246 (1954). - 4. JAYLE, M. F., Bull. Soc. Chim. biol. (Paris) 33, 876 (1951). - 5. Laureli, C. B. und Nrmann, M., Blood 12, 493 (1957). - 6. Connel, G. E. und O. Smrthies, Biochem. J. 72, 115 (1959). - 7. ZIJLSTRA, W. G., Klin. Wschr. 34, 384 (1956). - 8. Wibout, van LeUWEN und van der War, Organic analysis Vol. IV 14. New York (1960). - 9. KLUthe, R. und Mitarbeiter, in Vorbereitung. - 10. LACAPERE, J. BADIN, J. ReNier, J. Cl., WeIsendBACH, R. G. BASSET, Rev. Rheumat. XXIV. 691 (1957). - 11. JAYLE, M. F. und G. Boussier, Exp. ann. Biochem. med. 17, 157 (1955). - 12. Allison, A. C. und B. S. Blumberg, Arthrit. u. Rheum., V..1, 239 (1958). - 13. Kluthe, R. und W. MüLLER, Kongreßverhandlungen, X. Internat. Rheumatologen Kongreß, Rom 1961, S. 185. - 14. MülLER, W., R.
KLUTHE und H. MüLlER, Zschr. Rheumaforsch. Im Druck. 15. Kluthe, R. und P. Meyer, Klin. Wschr. 39, 205 (1961). 16. Kuuthe, R., Verh. Dtsch. Ges. inn. Med. 68, 561 (1962). 17: Troisier, J., Polonovsky, M., M. F. JAYLE und H. E. Brissaud, Bull. Acad. Med. Paris 123, 239 (1940). - 18. JAYLE, M. F., G. Boussier und M. J. Batias, C. R. Soc. Biol. Paris 14946 (1955). - 19. Kourilsky, R., S. Kourilsky und P. H. ChanteRENNE, Sem. hôp. Paris 25, 3416 (1949). - 20. Kluthe, R. und H. Sarre, Med. Klin. 57, 919 (1962). - 21. JayLE, M. F. und J. Vallin, Sem. hôp. Paris 28, 3133 (1952). - 22. Owen, J. A., J. R. Mc. KAY und C. Gor, Brit. Med. J. 1454 (1959). - 23. Schubothe, H. und H. J. KähLER, Die Hämoglobinurien. Handbuch d. ges. Hämatologie, 2. Aufl., Bd. 3, Urban u. Schwarzenberg, Berlin (1960) S. 638. - 24. NymanN, M., K. Gydell und B. Nossinn, Clin. Chim. Acta 4, 82 (1959).

Dr. med. Reinhold Kluthe Medizinische Klinik der Universität Freiburg 78 Freiburg i. Br., Hermann-Herder-Str. 6 